\title{
Reading on smartphone causes sigh inhibition with brain overactivity and comprehension decline
}

Motoyasu Honma ( $\square$ mhonma@med.showa-u.ac.jp)

Showa University School of Medicine https://orcid.org/0000-0003-4612-0115

\section{Yuri Masaoka}

Showa University School of Medicine

Natsuko lizuka

Showa University School of Medicine

\section{Sayaka Wada}

Showa University School of Medicine

\section{Sawa Kamimura}

Showa University School of Medicine

\section{Akira Yoshikawa}

Showa University School of Medicine

\section{Rika Moriya}

Showa University School of Medicine

\section{Shotaro Kamijo}

Showa University School of Medicine

Masahiko Izumizaki

Showa University School of Medicine

\section{Article}

Keywords: cognitive function, smartphones, reading comprehension

Posted Date: March 23rd, 2021

DOl: https://doi.org/10.21203/rs.3.rs-333878/v1

License: (c) (1) This work is licensed under a Creative Commons Attribution 4.0 International License.

Read Full License 


\section{Abstract}

Electronic devices have become an indispensable part of our lives, while negative aspects have been reported. One downside is that reading comprehension is reduced when reading from electronic device, but the cause of this is unclear. In this study, we investigated a cause for the decline in comprehension by simultaneously measuring respiration and brain activity during reading from a smartphone medium, on thirty-four healthy individuals. Here we found that, compared to a paper medium, reading from the smartphone elicits a suppression of sigh, brain overactivity in prefrontal cortex, and decline in reading comprehension. Furthermore, a path analysis suggests that interactive relationship between sigh inhibition and overactivity in prefrontal cortex causes the comprehension decline. These findings provide new insight into a respiration-mediated mechanism of cognitive function.

\section{Introduction}

In recent years, reading and studying on electronic devices has become more common. Although electronic devices have benefited mankind tremendously, they cause eyestrain and headaches ${ }^{1,2}$, and lead to poor reading comprehension ${ }^{3,4}$. This decline in comprehension with electric device might be due to poor concentration level or different sensory processing which might be associated with physiological state including brain and physiological activity level. Even if the content of the text is the same, the reading comprehension may be different depending on the visual environment. In a mechanism of sensory integration or cross-modality, vision has a dominant influence on the other senses ${ }^{5,6}$. Visual input might affect a brain state and physiological condition like a respiration.

Respiratory rhythm generator exists in the pacemaker neurons in the brainstem to maintain the homeostasis of the body ${ }^{7}$. When the brain senses that it is not getting enough oxygen, it makes it sigh. By sighing deeply and taking in oxygen, it can calm a heightened sympathetic nervous system and relieve stress $^{8}$. If the information that causes a sigh does not transmit to brain in one particular visual environment, stress may be not relieved. Furthermore, brain function may remain low, which may result in low cognitive performance. Indeed, respiration is affected by factors such as anxiety or stress, which change the depth and rhythm of breathing 9,10 . On the contrary, respiratory patterns affect the function of the frontal cortex and hippocampus, and therefore the formation of memories ${ }^{11-13}$. Attention to breathing also has been shown to elevate memory performance ${ }^{14}$. Thus, sigh is interactively associated with cortical factors, that is, sigh affects and is affected the state of brain. It is highly likely that cognitive performance is affected by visual environment via interactive relationship between brain and respiratory activity. This study examined the effects of smartphone device use on reading comprehension by measuring brain and respiratory activity simultaneously, on thirty-four healthy individuals.

\section{Results}


To investigate causes for reading comprehension decline with electronic devices, we prepared a repeatedmeasured design consisting of four conditions involving combinations of two mediums (smartphone and paper) and two sentences extracted from different texts (novels A and B; see Supplementary Text). One trial consisted of four sessions: resting state before reading, reading, resting state after reading, and reading test (Fig. 1a). Frontal brain activity (2 channels) were measured by functional Near-Infrared Spectroscopy (NIRS), and respiration activity ( 6 indexes) and metabolism patterns ( 2 indexes) were measured by respiratory Aeromonitor (see Methods).

For the reading score, repeated measured analysis of variance (RM-ANOVA) showed that reading medium affected reading scores $\left(F_{1,64}=27.367, P<0.0001, \eta^{2}=0.297\right)$, whilst the main effects of sentence and the interaction were not. Post-hoc tests revealed that scores with the paper medium were higher than that with smartphone medium, in both novel sentences $A$ and $B$ (respectively $P<0.05$, Fig. 1b). No main or interaction effects of medium or novel sentence were observed for duration of reading (Fig. 1c) or viewing distance between participants' eyes and the device (Fig. 1d).

Out of 6 respiratory and 2 metabolic patterns that we measured, the tidal volume was decreased during reading compared to after and before reading sessions, and sighs were increased during reading with paper medium (Fig. 2a). RM-ANOVA showed that there were an effect of session but not medium and interaction for the tidal volume (Fig. $2 b$, session: $F_{2,132}=34.145, P<0.0001, \eta^{2}=0.341$ ), inspiratory time (Fig. 2c, session: $F_{2,132}=8.639, P<0.0001, \eta^{2}=0.116$ ), expiratory time (Fig. $2 d$, session: $F_{2,132}=33.092$, $P<0.0001, \eta^{2}=0.334$ ), respiratory frequency (Fig. 2 e, session: $F_{2,132}=29.018, P<0.0001, \eta^{2}=0.305$ ), while there was an effect of session and interaction but not medium for the number of sighs (Fig. 2f, session: $F_{2,132}=20.530, P<0.0001, \eta^{2}=0.237$; interaction: $\left.F_{2,132}=9.169, P<0.0001, \eta^{2}=0.122\right)$. Posthoc tests revealed that regardless of the medium, tidal volume, inspiratory time, and expiratory time were reduced during reading compared to resting states, whilst respiratory frequency was increased (all $P<$ 0.05). Number of sighs was greater during reading with paper compared to smartphone (all $P<0.05$ ). No changes in minute ventilation, $\mathrm{O}_{2}$ consumption, and end tidal $\mathrm{CO}_{2}$ were confirmed through all the session (Fig. 2g-i).

We found that activity recorded with NIRS increased during reading compared to before and after reading sessions, and was increased during reading from the smartphone medium, compared to the paper medium (Fig. 3a). The RM-ANOVA showed that no main effect of medium and interaction in channel 1 (left probe), while a main effect of session was significant $\left(F_{2,132}=19.612, P<0.0001, \eta^{2}=0.229\right)$. In the channel 2 (right probe), there were no main effect of medium and interaction, while a main effect of session was observed $\left(F_{2,132}=10.430, P<0.0001, \eta^{2}=0.136\right)$. Post-hoc tests revealed that the activity was increased during reading compared to resting states on both the left $(P<0.05$, Fig. 3b) and right $(P<$ 0.05 , Fig. $3 c$ ) probes. Furthermore, the activity when reading from the smartphone was higher than when reading from paper on the left probe (all $P<0.05$ ). 
We conducted a path analysis to examine a mechanistic route for results of the reading tests. Six respiratory indexes, two metabolic indexes, two NIRS indexes, and reading score were set as the observed variables. In the most suitable model (Fig. 4 , the goodness of fit index $=0.881$ ), there are a covariate relationship between left NIRS channel activity and the number of sighs $(P=0.021)$, and a direct relationship between the right NIRS channel activity and its impact on reading score $(P=0.003)$.

\section{Discussion}

The current study replicated the previously reported lower reading performance with the smartphone medium compared to the paper medium ${ }^{3,4}$. Respiratory results show that breathing becomes shallow and fast during reading regardless of medium, and number of sighs is reduced during reading from a smartphone. Shallow and fast breathing may cause decreased respiratory variability, with sighs functioning to reset this ${ }^{15,16}$. Reading on a smartphone may therefore cause inhibition of sighs. For NIRS activity, if brain functions were common with respect to syntactic processing during reading in both paper and smartphone, then brain activity in prefrontal cortex with the smartphone may be overactive. That activity selectively on the left side may reflect the predominant activity of the left hemisphere for processing reading ${ }^{17,18}$. Furthermore, the path analysis suggests that lower reading score results from the interaction between brain and respiration activities.

In physiologically, sigh increases for response for physiological stress such as hypoxia and hypercapnia by stimulation of the peripheral chemoreceptors ${ }^{19,20}$. Specific neuronal subsets influence the respiratory rhythm generator, the pre-Bötzinger complex, involved for sigh circuit for physiological purpose, and also for emotional factors ${ }^{8}$. No changes in metabolism $\left(\mathrm{O}_{2}\right.$ consumption and end tidal $\left.\mathrm{CO}_{2}\right)$ as well as constant minutes ventilation in our study suggests that cause of sigh generation might be associated with more higher cortical factors.

Deeper understanding may result from new information being deeply connected to many memories by attentional function ${ }^{21-23}$. Working memory training also improves reading comprehension ${ }^{24}$. Furthermore, attention and breathing functions share a common center in the locus coeruleus in the brain ${ }^{25,26}$. Respiration affects the frontal cortex and hippocampus during memory formation ${ }^{11-13}$. Attention to breathing is reported to increased memory performance ${ }^{14}$. Thus, prefrontal cortex and respiratory activity like sigh are closely related, and the interactive relationship may directly impact cognitive function.

We propose a mechanism of reading behavior processing that combines brain and respiratory activities (Supplemental Fig. 1). Intrinsically photosensitive retinal ganglion cells (ipRGCs) that respond to blue light project to various brain regions such as perihabenular nucleus that modulate arousal, stress, and learning ${ }^{27}$. Blue light also causes higher sustained attention compared to green light ${ }^{28}$. The results of smartphone medium may be caused by a stress in ipRGCs activity due to the blue light. Generally, increased activity in prefrontal cortex suggests that the brain was under a constant stress, while 
overactivity in prefrontal cortex suggests that the brain was under an intense stress. With the paper medium, moderate stress may generate sighs (or deep breaths), and appears to restore respiratory variability and control of prefrontal brain activity. In contrast, with the smartphone medium, a force from blue light may be required to sustain task attention, and intense stress may inhibit generation of sighs, causing overactivity in the prefrontal cortex. Indeed, sighing is associated with attention and stress, and it resets the respiratory variability ${ }^{15,29,30}$, and the reset may be associated with improved executive functions such as cognition and motivation.

This study provides a suggestion that reduced reading comprehension on smartphone devices may be caused by reduced sighing with overactivity in prefrontal cortex, although the effect on electronic devices other than smartphones is unconfirmed. Since sighs, whether voluntary or involuntary, have a regulating effect on disordered breathing ${ }^{32}$, it may be beneficial to take deep breaths whilst reading.

\section{Methods}

Participants. This study was approved by the ethics committee of Showa University School of Medicine and conducted according to the principles of the Declaration of Helsinki (trial identifier number: 2179). Thirty-four Japanese university students provided written informed consent prior to the experiments. All participants were right-hand dominant and had no history of neurological or psychiatric disease (20 females; mean age $=20.4$, S.D. $=0.8$ ). Participants had normal vision with $/$ without correction .

Experimental design and setting. To examine the influence of using a smartphone on reading, we set up four conditions involving combinations of two mediums (smartphone and paper) and two sentences (novels A and B; see Supplementary Text) (Fig. 1A). Participants conducted two trials for each different condition to avoid duplication of medium and sentence conditions (for example, if the first trial was novel A on smartphone, second trial was novel B on paper). A trial consisted of four sessions; resting state before reading, during reading, resting state after reading, and reading test. Participants were made to sit down and their torso and arms were secured. They were asked to read the allocated sentence on the allocated medium in the reading session. During resting state sessions, participants were asked to open their eyes and look at a wall. After this, they took a reading test consisting of 10 questions related to the contents of the sentences (Supplementary text 1). They were also asked to breathe through their nose during all sessions. There was no limit to reading time and 2 min was spent in the resting state before/after reading. At the end of the experiment, participants were asked whether they had read the novels used in the experiment in the past. All participants answered that they had never read the novels.

The observation distance was determined by the convenience of the participants, and the experimenter measured the distance between the device and the eyes of the participants. The factors of medium and sentence had no influence on reading time or viewing distance (Extended data Fig. 1). Each sentence originated from a passage one of two novels written by the same author Haruki Murakami (Novel A: Norwegian Wood, 1987, 3060 Japanese characters; or Novel B: Colorless Tsukuru Tazaki and His Years of Pilgrimage, 2013, 3067 Japanese characters) (Supplementary text 1). The panel size of the smartphone 
was 5.0 inches (resolution pixel: height: 1920, width: 1080) and a text size was 0.85 degrees. The text size of paper medium was identical to the smartphone device. In addition, weight (148 gram) and outer frame (height: $144 \mathrm{~mm}$, width: $72 \mathrm{~mm}$, thickness: $8.6 \mathrm{~mm}$ ) of the paper medium was also identical to the smartphone device by using a pad board.

The participant wore two functional Near-Infrared Spectroscopy (NIRS) probes (Hb13-2, ASTEM, Kanagawa, Japan) for measurement of brain activity in the prefrontal cortex ${ }^{33,34}$, and a facemask with a transducer connected to a respiratory monitor (Aeromonitor AE280, Minato Medical Science, Osaka, Japan) for measurement of respiratory patterns and metabolism ${ }^{35,36}$. The NIRS calculated oxygenated hemoglobin $(\mathrm{Oxy}-\mathrm{Hb})$ concentration $[\mathrm{mM}]$ at two places on the forehead. The respiratory monitor calculated 6 indexes of the respiratory pattern: tidal volume (depth of breathing, [ml]), respiratory frequency (number of breaths per minute, [n/min]), inspiratory time (duration to inhale breath, [s]), expiratory time (duration to exhale breath, [s]), minute ventilation (ventilation rate per minute, [I]), number of sighs (deep breathing, [n]), and 2 indexes of the metabolic pattern $\left(\mathrm{O}_{2}\right.$ consumption [ml], End tidal $\mathrm{CO}_{2}$ $[\%])$. A sigh was defined as at least twice the average tidal volume for each session ${ }^{37-39}$.

Statistics. Two-way analysis of variance (ANOVA) and post-hoc t-tests with Bonferroni correction were performed for testing of the main effects and interactions of medium (smartphone and paper) and novel sentence (novel sentences A and B). Repeated-measures ANOVA (RM-ANOVA) and post-hoc tests with Bonferroni correction were performed for medium and session conditions (before, during, and after reading). All tests were two-tailed. Results are presented as mean \pm standard error of mean (SEM) and effect size (eta squared, $\eta^{2}$ ). SPSS 24.0 (IBM, Inc., Chicago, IL) was used for ANOVA and RM-ANOVA. Relationships among the 6 respiration indexes, 2 metabolism indexes, 2 NIRS indexes, and the comprehension score were calculated by path analysis. The goodness of fit index was determined. AMOS 27.0 was used for path analysis. The statistical significance criterion was defined as adjusted $P<0.05$.

\section{Declarations}

\section{Data availability}

The data that support the findings of this study are available from the corresponding author upon request. Source data and Supplementary information are provided with the paper.

\section{Code availability}

Analyses used in this study are largely standard approaches for this type of data. The code that supports these findings is available upon request from the corresponding author.

\section{Acknowledgments}

We thank M. lizuka for experiment monitoring. This work was supported by JSPS KAKENHI Grant Number JP18K03185. 


\section{Author contributions}

M.H. conceptualized the experiment and wrote the original manuscript. Y.M., N.I., and S.W. performed the experiment. M.H., Y.M., S.K., A.Y., S.K., R.M., and I.M. performed analysis. M.H. and Y.M. conducted the review of manuscript. All the authors participated in editing the manuscript.

\section{Competing interests}

The authors declare no competing interests.

\section{Additional information}

Source data is available for this paper at...

Supplementary information is available for this paper at...

Correspondence and requests for materials should be addressed to M.H.

\section{References}

1. Jaiswal, S. et al. Ocular and visual discomfort associated with smartphones, tablets and computers: what we do and do not know. Clin Exp Optom 102, 463-477, doi:10.1111/cxo.12851 (2019).

2. Golebiowski, B. et al. Smartphone Use and Effects on Tear Film, Blinking and Binocular Vision. Curr Eye Res 45, 428-434, doi:10.1080/02713683.2019.1663542 (2020).

3. Wästlund, E. Reinikka, H. Norlander, T. \& Archer, T. Effects of VDT and paper presentation on consumption and production of information: Psychological and physiological factors. Comput Human Behav 21, 377-394 (2005).

4. Kang, Y. Y., Wang, M. J. J. \& Lin, R. Usability evaluation of E-books. Displays 30, 49-52 (2009).

5. Driver, J. \& Spence, C. Cross-modal links in spatial attention. Philos Trans R Soc Lond B Biol Sci 353, 1319-1331, doi:10.1098/rstb.1998.0286 (1998).

6. Honma, M. et al. Impairment of cross-modality of vision and olfaction in Parkinson disease. Neurology 90, e977-e984, doi:10.1212/WNL.0000000000005110 (2018).

7. Feldman, J. L., \& Del Negro, C. A. Looking for inspiration: new perspectives on respiratory rhythm. Nat Rev Neurosci 7, 232-242 (2006).

8. Li, P. et al. The peptidergic control circuit for sighing. Nature 530, 293-297, doi:10.1038/nature16964 (2016).

9. Masaoka, Y., Onaka, Y., Shimizu, Y., Sakurai, S. \& Homma, I. State anxiety dependent on perspiration during mental stress and deep inspiration. J Physiol Sci 57, 121-126, doi:10.2170/physiolsci.RP000607 (2007).

10. Jerath, R. \& Beveridge, C. Respiratory Rhythm, Autonomic Modulation, and the Spectrum of Emotions: The Future of Emotion Recognition and Modulation. Front Psychol 11, 1980 (2020). 
11. Heck, D. H., Kozma, R. \& Kay, L. M. The rhythm of memory: how breathing shapes memory function. J Neurophysio/ 122, 563-571, doi:10.1152/jn.00200.2019 (2019).

12. Zelano, C. et al. Nasal Respiration Entrains Human Limbic Oscillations and Modulates Cognitive Function. J Neurosci 36, 12448-12467, doi:10.1523/JNEUROSCI.2586-16.2016 (2016).

13. Biskamp, J., Bartos, M. \& Sauer, J. F. Organization of prefrontal network activity by respiration-related oscillations. Sci Rep 7, 45508, doi:10.1038/srep45508 (2017).

14. Fujino, M., Ueda, Y., Mizuhara, H., Saiki, J. \& Nomura, M. Open monitoring meditation reduces the involvement of brain regions related to memory function. Sci Rep 8, 9968, doi:10.1038/s41598-01828274-4 (2018).

15. Vlemincx, E. et al. Respiratory variability and sighing: a psychophysiological reset model. Biol Psycho/ 93, 24-32, doi:10.1016/j.biopsycho.2012.12.001 (2013).

16. Ramirez, J. M. The integrative role of the sigh in psychology, physiology, pathology, and neurobiology. Prog Brain Res 209, 91-129, doi:10.1016/B978-0-444-63274-6.00006-0 (2014).

17. Wang, K. et al. Left posterior prefrontal regions support domain-general executive processes needed for both reading and math. J Neuropsycho/ 14, 467-495, doi:10.1111/jnp.12201 (2020).

18. Patael, S. Z. et al. Brain basis of cognitive resilience: Prefrontal cortex predicts better reading comprehension in relation to decoding. PLoS One 13, e0198791, doi:10.1371/journal.pone.0198791 (2018).

19. Glogowska, M., Richardson, P. S., Widdicombe, J. G. \& Winning, A. J. The role of the vagus nerves, peripheral chemoreceptors and other afferent pathways in the genesis of augmented breaths in cats and rabbits. Respir Physio/ 16, 179-196 (1972).

20. Cherniack, N. S., von Euler, C., Głogowska M. \& Homma, I. Characteristics and rate of occurrence of spontaneous and provoked augmented breaths. Acta Physiol Scand 111, 349-360 (1981).

21. Karakas, S. A review of theta oscillation and its functional correlates. Int J Psychophysiol, doi:10.1016/j.jpsycho.2020.04.008 (2020).

22. Spanoudis, G. \& Demetriou, A. Mapping Mind-Brain Development: Towards a Comprehensive Theory. J Intel/ 8, doi:10.3390/jintelligence8020019 (2020).

23. Palacio, N. \& Cardenas, F. A systematic review of brain functional connectivity patterns involved in episodic and semantic memory. Rev Neurosci 30, 889-902, doi:10.1515/revneuro-2018-0117 (2019).

24. Chein, J. M. \& Morrison, A. B. Expanding the mind's workspace: training and transfer effects with a complex working memory span task. Psychon Bull Rev 17, 193-199, doi:10.3758/PBR.17.2.193 (2010).

25. Melnychuk, M. C. et al. Coupling of respiration and attention via the locus coeruleus: Effects of meditation and pranayama. Psychophysiology 55, e13091, doi:10.1111/psyp.13091 (2018).

26. Doll, A. et al. Mindful attention to breath regulates emotions via increased amygdala-prefrontal cortex connectivity. Neuroimage 134, 305-313, doi:10.1016/j.neuroimage.2016.03.041 (2016). 
27. Fernandez, D. C. et al. Light Affects Mood and Learning through Distinct Retina-Brain Pathways. Cell 175, 71-84 (2018).

28. Lockley, S. W. et al. Short-wavelength sensitivity for the direct effects of light on alertness, vigilance, and the waking electroencephalogram in humans. Sleep 29, 161-168 (2006).

29. Vlemincx, E. et al. Why do you sigh? Sigh rate during induced stress and relief. Psychophysiology 46, 1005-1013, doi:10.1111/j.1469-8986.2009.00842.x (2009).

30. Grassmann, M., Vlemincx, E., von Leupoldt, A., Mittelstadt, J. M. \& Van den Bergh, O. Respiratory Changes in Response to Cognitive Load: A Systematic Review. Neural Plast 2016, 8146809, doi:10.1155/2016/8146809 (2016).

31. Kimhy, D. et al. The association of cardiac vagal control and executive functioning-findings from the MIDUS study. J Psychiatr Res 47, 628-635, doi:10.1016/j.jpsychires.2013.01.018 (2013).

32. Vlemincx, E., Taelman, J., Van Diest, I. \& Van den Bergh, O. Take a deep breath: the relief effect of spontaneous and instructed sighs. Physiol Behav 101, 67-73, doi:10.1016/j.physbeh.2010.04.015 (2010).

33. Kanayama, N. \& Niwayama, M. Examiner's finger-mounted fetal tissue J Biomed Opt 19, 067008, doi: 10.1117/1.JBO.19.6.067008 (2014).

34. Soltanlou, M., Sitnikova, M. A., Nuerk, H. C. \& Dresler, T. Applications of Functional Near-Infrared Spectroscopy (fNIRS) in Studying Cognitive Development: The Case of Mathematics and Language. Front Psycho/ 9, 277, doi: 10.3389/fpsyg.2018.00277 (2018).

35. Masaoka, Y., Koiwa, N. \& Homma, I. Inspiratory phase-locked alpha oscillation in human olfaction: source generators estimated by a dipole tracing method. J Physio/ 566, 979-997, doi:10.1113/jphysiol.2005.086124 (2005).

36. Masaoka, Y., Sugiyama, H., Katayama, A., Kashiwagi, M., Homma, I. Slow breathing and emotions associated with odor-induced autobiographical memories. Chem Senses 37, 379-388. doi: 10.1093/chemse/bjr120 (2012).

37. Vlemincx, E., Meulders, M. \& Luminet, O. A sigh of relief or a sigh of expected relief: Sigh rate in response to dyspnea relief. Psychophysiology 55, doi:10.1111/psyp.12979 (2018).

38. Vlemincx, E., Van Diest, I. \& Van den Bergh, O. A sigh following sustained attention and mental stress: effects on respiratory variability. Physiol Behav 107, 1-6, doi:10.1016/j.physbeh.2012.05.013 (2012).

39. Vlemincx, E., Van Diest, I. \& Van den Bergh, O. A sigh of relief or a sigh to relieve: The psychological and physiological relief effect of deep breaths. Physiol Behav 165, 127-135, doi:10.1016/j.physbeh.2016.07.004 (2016).

\section{Figures}




\section{a}
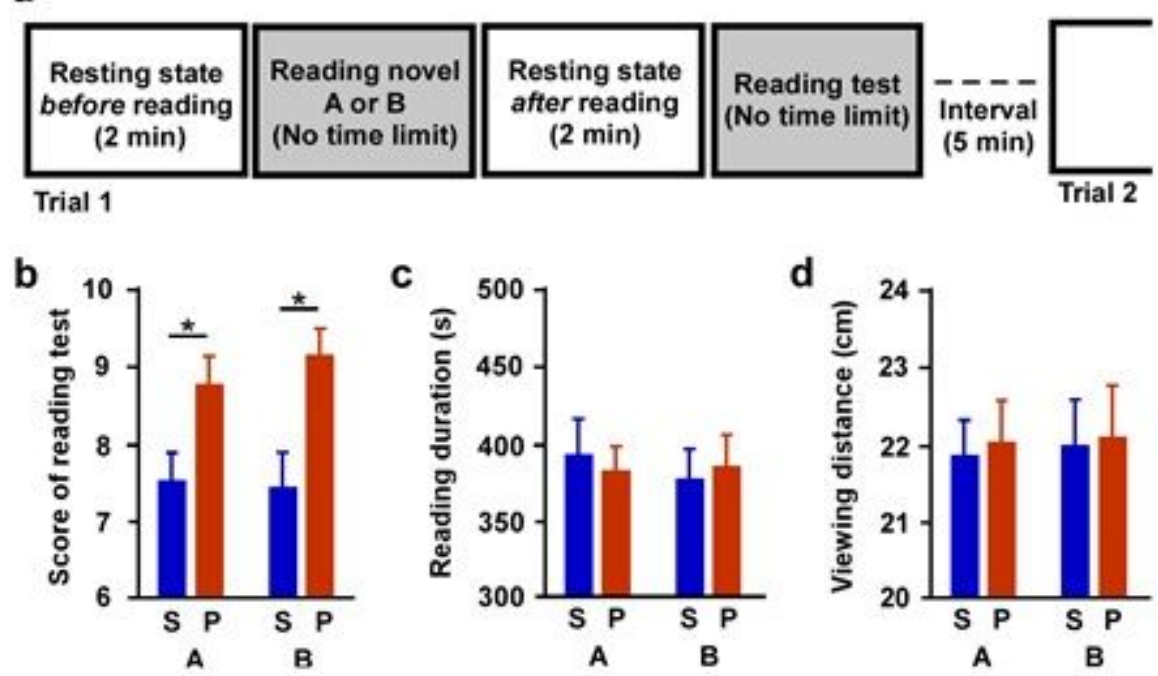

\section{Figure 1}

Reading score is increased with the paper medium. a, Participants conducted two trials in each different condition. A trial consisted of four sessions; resting state before reading, reading, resting state after reading, and reading test. Reading time was not limited, and 2 min was spent in the resting state before/after reading. b, For reading test score, ANOVA showed that the main effect of medium was significant, while the main effects of sentence and the interaction were not. Score with the smartphone medium was lower than that with the paper medium in sentences from both novel $A$ and $B\left({ }^{*} P<0.05\right)$. $c, d, A N O V A$ showed that no main and interaction effect of medium and novel sentence in both the duration of reading session and the viewing distance between participants eyes and device. Error bars show standard error of the mean. S: smartphone, P: paper, A: novel sentence A, B: novel sentence B. 

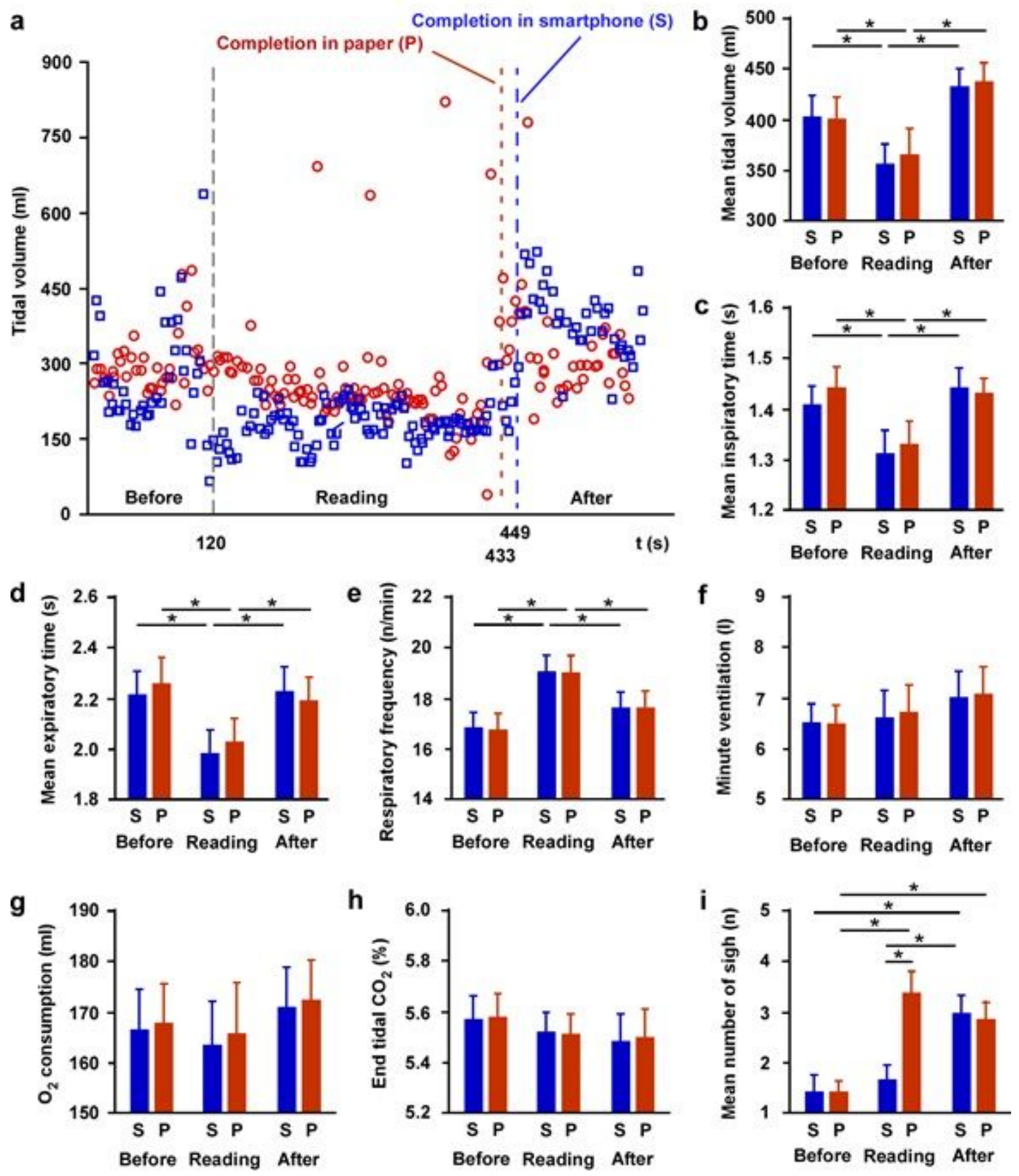

Figure 2

Sighs are inhibited during reading with the smartphone medium. a, A representative pattern of the tidal volume and sighs during the before, during, and after reading sessions. b-d, RM-ANOVA showed that, regardless of medium, tidal volume, inspiratory time, and expiratory time were reduced during the reading sessions ( $\left.{ }^{*} P<0.05\right)$. e, Respiratory frequency was increased during reading sessions $\left({ }^{*} P<0.05\right) . f-h$, There were no difference between mediums in the minute ventilation, $\mathrm{O} 2$ consumption, and End tidal $\mathrm{CO} 2$, and there were no change throughout the sessions. i, Number of sighs was increased during the reading session with the paper medium, and was increased in the after reading session with both medium $\left({ }^{*} \mathrm{P}<\right.$ 0.05). Error bars show standard error of the mean. S: smartphone, P: paper. 

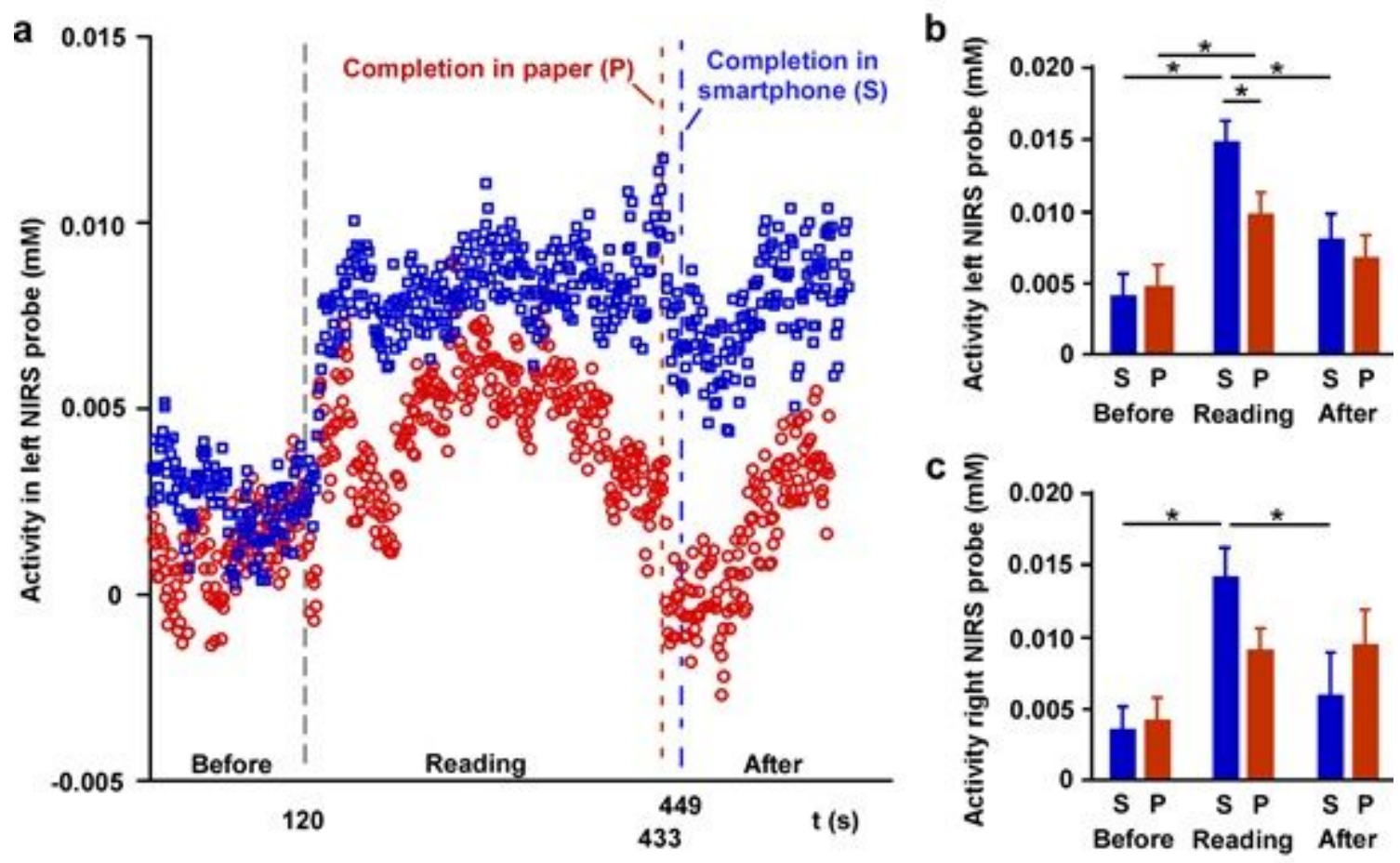

Figure 3

Brain activity around the forehead is increased during reading with the smartphone medium. a, A representative pattern of NIRS activity on the left probe during the before, during, and after reading sessions. b,c, RM-ANOVA showed that activity recorded with NIRS was increased during the reading sessions with the smartphone medium from the left and right probes compared to the before and after reading sessions. The difference between the mediums was clearly visible in the left probe $\left({ }^{\star} P<0.05\right)$. Error bars show standard error of the mean. S: smartphone, P: paper.

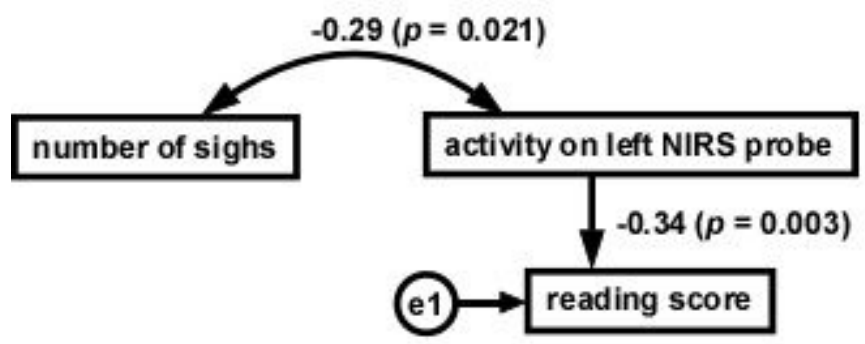

Figure 4

Route to reading score in path analysis. Six respiratory indexes (minute ventilation, tidal volume, respiratory frequency, inspiratory time, expiratory time, and number of sighs), two metabolic patterns (02 consumption and End tidal CO2), two NIRS indexes (right/left) in the reading sessions, and reading score were set as the observed variables. The analysis showed a relationship between number of sighs and left NIRS activity, and that reading score was directly influenced by left NIRS activity. Numbers mean standardized path coefficients. 


\section{Supplementary Files}

This is a list of supplementary files associated with this preprint. Click to download.

- SourceDataCommBiol.xlsx

- SupplementaryInformationforCommBiol.pdf 